\title{
Approaches to determining the kinetics for the formation of a nano-dispersed substance from the experimental distribution functions of its nanoparticle properties
}

\author{
S. Z. Adzhiev ${ }^{1}$, I. V. Melikhov ${ }^{1}$, V. V. Vedenyapin ${ }^{2}$ \\ ${ }^{1}$ Lomonosov Moscow State University, Moscow, Russia \\ ${ }^{2}$ Keldysh Institute of Applied Mathematics of Russian Academy of Sciences; RUDN-University, Moscow, Russia \\ sergeyadzhiev@yandex.ru, vicveden@yahoo.com, melikhov@ radio.chem.msu.ru
}

DOI 10.17586/2220-8054-2019-10-5-549-563

\begin{abstract}
In the present paper, we discuss the kinetic equations for the evolution of particles of a nanodispersed substance, distinguishing by properties (sizes, velocities, positions, etc.). The aim of the present investigation is to determine the coefficients for the equations by the distribution functions, which are obtained experimentally. The experiment is characterized by the time interval, which is needed for the measurement of the distribution function. However, the nanodispersed substance is obtained from a highly supersaturated solution or vapor and this time interval is large, thus, one is able to measure distribution functions only when the processes of the integration and the fragmentation of the particles become rather slow. So it is advisable to reconstruct the kinetics for the formation of a nanodispersed substance by the experimental distribution functions measured when the processes are rather slow. The first problem that arises is the obtaining of correct equations, and, hence, the derivation of the equations from each other. From the discrete system of equations for the evolution of discrete distribution functions of particles of a nanodispersed substance, we obtain the continuum equation of the Fokker-Planck type, or of the Einstein-Kolmogorov type, or of the diffuse approximation on the distribution function of nanoparticles distinguishing by the numbers of molecules forming them. We consider the distribution functions, which approximate the experimental data. We determine the coefficients for the equation of the Fokker-Planck type by the stationary and non-stationary distribution functions of a nanodispersed substance. Due to unity of the kinetic approach, the present work may be useful for specialists of various areas, who study the evolution of structures (not only with nanosize) with differing properties.
\end{abstract}

Keywords: nanodispersed substance, nanoparticles, distribution function of particles by properties, emerging and growth of nanoparticles, aggregation and agglomeration, the Fokker-Planck equation, the Smoluchowski system of equations, the Becker-Döring equations.

Received: 8 September 2019

Revised: 5 October 2019

\section{Statement of the problem}

The problem, which is solved, is the mathematical description of the kinetics of the emerging, growth and aggregation of nanoparticles by their experimental distribution functions by properties.

The nanodispersed substance is obtained from a highly supersaturated solution or vapor. Significant supersaturation is required to ensure that during formation the particles do not have time to grow to sizes larger than nanometers, the nanoparticles have emerged in sufficient quantity so that the stage of their aggregation becomes dominant. In this case, we obtain aggregates of nanoparticles, i.e. a nanodispersed substance.

The experiment is characterized by the time interval, which is needed for the measurement of the distribution function. It is possible to obtain it only if the variation of the distribution function during this time interval is negligible. So, we are able to measure distribution functions only when the processes of the integration and the fragmentation of the particles are rather slow. In the beginning of the process of the forming of a nanodispersed substance the supersaturation is large and we are not able to measure the distribution function. So we need to reconstruct the kinetics for the formation of a nanodispersed substance by the experimental distribution functions measured, when the process become rather slow. One measurement is not sufficient, we should make the set of experiments varying the parameters of system's condition: the concentration of initial particles (molecules) and the temperature, in order to obtain the coefficients for the equations depending on these parameters.

The main idea for mathematical description of the experimental results of, which determines the structure of the article, is as follows: first, the statement of the problem arises: the parameters of the distribution function are determined. At this stage, the experimenters suggest which parameters they can measure, i.e. which parameters to choose for the model. Then, the first step in mathematical modeling is to create an a priori model containing unknown functions as parameters, which should be determined later. The a priori model consists of writing the correct equations containing unknown parameters. The model should answer the following questions: what are the conditions of the experiment, how and in what range they should be varied and what should be determined in the experiment in order to obtain these unknown functions of the a priori model. On the basis of experiments, these unknown functions are determined, and the a posteriori model arises. Thus, the mathematical description of the experiments is obtained. After 
that, the investigation is supposed to be repeated, expanding the list and the intervals of variation of the conditions taken into account. The goal is to predict how to synthesize materials.

In the frameworks of a priori models in the papers of J. C. Maxwell [1], L. Boltzmann [2] and etc. the time dependent distribution function by velocities and coordinates appears. Now, there is a multi-parameter description, which is due to the fact that it becomes available for experimental observation. Currently, several methods have been developed for measuring the size distribution of nanoparticles: electron microscopy, the method of dynamic light scattering, e.g. [3,4]. The problem is to write the equations for the distribution function of particles (bodies) by these many parameters.

In the present paper, we continue the line of works [5-8] and others, devoted to mathematical modeling of emerging and growth of hierarchical structures of a dispersed substance, using the kinetic approach.

In [6], a model was proposed for nanostructure creation of as a result of growth of particles in a supersaturated medium with their multistage modification. The multistage modification was done in order to give particles the desired properties. The aggregation of nanoparticles in agglomerates and the integration of aggregates with each other was considered and the discrete and continuum balance equations for the number of particles and aggregates, taking into account the possibility of their growth and fragmentation, were formulated. The continuum equation has yielded to the evolutionary equation of the Fokker-Planck type, containing frequency functions. They describe the process of aggregate self-organization by the selection of more stable forms. Moreover, each stage of modification is characterized by its own frequency functions. They are subject to independent determination. Frequency functions for all types of impacts used for the creation of nanostructures can form the basis of a methodology for their optimal synthesis.

Typically, a nanostructure is a hierarchical structure. Molecules coalesce and give the original aggregates of molecules (clusters). Coalescences of original agglomerates are secondary aggregates. They also may coalesce with separate molecules. Similarly, the coalescence of secondary aggregates with each other give the tertiary agglomerates, etc. A particle of given generation (or type, kind) is the body in which the constituent elements of all lower generations can be identified. Types of bodies distinguishing by the levels of hierarchical structure (generation numbers) will be denoted by the parameter $k$ : the particles of each $k$-th generation become larger by attaching particles of all kinds with the parameter $j<k$, and the acts of coalescences randomly alternate with the acts of fragmentations. As a result, the growth process of the particles forms bodies of a dispersed substance, which have a hierarchical structure.

For description of particle formation, it is advisable to distinguish several essential stages of the process: first, original aggregates emerge and grow, and then the stage of aggregation or the formation of agglomerates of the original clusters as a result of their collisions with each other comes, and then the formation of their aggregates occurs. Although all these processes proceed at the same time, but under some conditions, first the process of formation of original particles dominates, then the process of formation of their aggregates (i.e. secondary agglomerates) prevails, then the process of formation of aggregates of secondary agglomerates (i.e. tertiary aggregates) is main, etc.

Under such conditions, the formation of dispersed substance occurs incrementally, and each stage contributes to the nanostructure of the substance.

In order to create a nanostructure (and, generally speaking, some kind of structure) with the desired properties, the phenomena of the emerging and the disruption of the original subnanoparticles, the growth and the dissolution, the appearance and the elimination of growth defects, the capture and the release of the captured impurities, the coalescence and the destruction of agglomerates, recrystallization and the topochemical reactions of sintering and the ceramization are used [5-9]. For describing these phenomena we take into account the properties $\mathbf{X} \equiv\left\{\mathbf{X}_{1}, \mathbf{X}_{2}\right\}$ of each particle: $\mathbf{X}_{1} \equiv\left\{x_{1}, x_{2}, \ldots, x_{i}, \ldots, x_{r}\right\}$ are external properties, and $\mathbf{X}_{2} \equiv\left\{x_{r+1}, x_{r+2}, \ldots, x_{i}, \ldots, x_{s}\right\}$ are internal ones. The external parameters of state of a particle characterize its material and energy interaction with the medium, and the internal ones characterize the redistribution of the matter and the energy inside the particle [9].

The state of each particle of any level of the hierarchical structure of a nanodispersed substance is characterized by many parameters. The state of each molecule is characterized by the following external parameters: mass (the volume), the coordinate and the velocity of its center of mass. In the case of aggregates of the first generation, it is supplemented by the next external parameters: parameters of the shape, the surface topography and the volume, which can be more than the sum of the volumes of molecules constituting the given aggregate (the porosity is possible). In the case of secondary agglomerates, it is complemented by the following internal parameters: the positions of the center of mass (relative to center of mass of the considered secondary aggregate) of each of the original aggregates and of the remaining individual molecules constituting it as well as the parameters which define the orientation of the original clusters composing the given secondary agglomerate.

At all stages of the process, the considered system is heterogeneous, and there are the molecules, aggregates of molecules, growing by joining molecules, aggregates of second generation, etc. at its every area. As a first approximation it is appropriate to accept that the mixer provides homogeneity for the medium and the substance forming the 
phase. In the continuum approximation, each part of the system is characterized by its distribution function of particles by properties: $\Psi(\mathbf{X}, t)=\sum_{k} \Psi_{k}(\mathbf{X}, t), N_{k, \mathbf{X}}=\int_{\mathbf{X}_{0}}^{\mathbf{X}} \Psi_{k}\left(\mathbf{X}^{\prime}, t\right) d \mathbf{X}^{\prime}$. Here, $\Psi_{k}(\mathbf{X}, t)$ is a distribution function of particles of the generation $k, N_{k, \mathbf{X}}$ is a number of particles of the type $k$ in the system, for which the state parameters are smaller than the corresponding component of the vector $\mathbf{X}, \mathbf{X}_{0}$ is the minimum possible value of property $\mathbf{X}$. $\mathbf{X}$ does not include the spatial coordinates of the particle, which are identified with the coordinates of the area of the system. The distribution functions by these properties $\mathbf{X}$ depends upon the properties of the medium enveloping the particles.

The main parameter of the distribution function of particles of each of $k$ kinds is defined as the first parameter in the decomposition below, and it is advisable to take the number of molecules $n$ constituting the particle as the main parameter: $x_{1}=n$, and then:

$$
\Psi_{k}(\mathbf{X}, t)=\psi_{k}(n, t) \cdot f_{k, 2}\left(x_{2} \mid n ; t\right) \cdot \ldots \cdot f_{k, s}\left(x_{s} \mid n, x_{2}, \ldots, x_{s-1} ; t\right),
$$

where $\psi_{k}(n, t)$ is the distribution function of particles by the number of molecules which constitute the particle, $f_{k, i}\left(x_{i} \mid n, x_{2}, \ldots, x_{i-1} ; t\right)$, where $i=2, \ldots, s$, are the densities of distributions of particles by the property $x_{i}$ under the condition that the parameters, which are written after, are fixed: $\int f_{k, i}\left(x_{i} \mid n, x_{2}, \ldots, x_{i-1} ; t\right) d x_{i}=1$.

The formula (1.1) is sufficient to prove for the case of two variables. Then it is proved by induction.

Lemma. Let a function $f\left(x_{1}, x_{2}, \ldots, x_{s}\right)>0$ is such, that $\int f\left(x_{1}, \ldots, x_{s}\right) d x_{s-k} \ldots d x_{s}(k=0, \ldots, s-2)$ is finite. Then the function $f$ may be represented in the unique way as follows:

$$
f\left(x_{1}, x_{2}, \ldots, x_{s}\right)=g_{1}\left(x_{1}\right) g_{2}\left(x_{1}, x_{2}\right) \times \ldots \times g_{s}\left(x_{1}, x_{2}, \ldots, x_{s}\right),
$$

where

$$
\int g_{i}\left(x_{1}, x_{2}, \ldots x_{i}\right) d x_{i}=1 \text {. }
$$

The proof. According to the method of mathematical induction, this formula is sufficient to prove for the case of two variables. Functions $g_{1}\left(x_{1}\right) \equiv \int f\left(x_{1}, x_{2}\right) d x_{2}, g_{2}\left(x_{1}, x_{2}\right) \equiv \frac{f\left(x_{1}, x_{2}\right)}{g_{1}\left(x_{1}\right)}$ satisfy equality $(1.2): f\left(x_{1}, x_{2}\right)=$ $g_{1}\left(x_{1}\right) g_{2}\left(x_{1}, x_{2}\right)$, with condition (1.3): $\int g_{2}\left(x_{1}, x_{2}\right) d x_{2}=1$.

This representation leads to the description of the probability theory for the second variable $x_{2}$ as a random variable: $g_{2}\left(x_{1}, x_{2}\right)$, which is a distribution function of the $x_{2}$ under the condition $x_{1}$, and is written in probability theory as $g_{2}\left(x_{2} \mid x_{1}\right)$.

The following formula in probability theory called Bayes formula [10], and the kinetic theory makes sense of the reduced description, as noted in [5].

Example 1 [1,2]. If a variable $\mathbf{v}$ is considered as a probabilistic one, and the description is reduced as a result of collisions to the locally Maxwell distribution:

$$
f(\mathbf{x}, \mathbf{v}, t)=n(\mathbf{x}, t)\left(\frac{M}{2 \pi \kappa_{B} T(x, t)}\right)^{3 / 2} \exp \left(-\frac{M(\mathbf{v}-\mathbf{V}(\mathbf{x}, t))^{2}}{2 \kappa_{B} T(\mathbf{x}, t)}\right),
$$

where $f(\mathbf{x}, \mathbf{v}, t)$ is a distribution functions of molecules with mass $M$ by space $\mathbf{x} \in R^{3}$, velocities $\mathbf{v} \in R^{3}$ at a time $t$. Here, $n(\mathbf{x}, t) \equiv \psi_{1}(\mathbf{x}, t)$ is a concentration of such particles with mass $M, T(\mathbf{x}, t)$ is the temperature, $\kappa_{B}$ is the Boltzmann constant, $\mathbf{V}(\mathbf{x}, t)$ is the value of the average velocity of particles with mass $M$.

The example 1 we consider as a classical one. Let us consider another example of distribution (1.1) from [11].

Example 2. [1,2,11]. An example of distribution (1.1) is given by the Maxwell distribution function of particles with mass $M_{n}=n M_{0}$, where $M_{0}$ is the mass of one molecule of a substance forming the solid phase, $n$ is a number of molecules constituting the particle [11]:

$$
\Psi_{1}(n, \mathbf{x}, \mathbf{p}, t)=\psi_{1}(n, \mathbf{x}, t) \times \frac{1}{\left(2 \pi M_{n} \kappa_{B} T(\mathbf{x}, t)\right)^{3 / 2}} \exp \left(-\frac{\left(\mathbf{p}-M_{n} \mathbf{V}(\mathbf{x}, t)\right)^{2}}{2 M_{n} \kappa_{B} T(\mathbf{x}, t)}\right),
$$

where $\mathbf{p}$ is a momentum of a the particle with the mass $M_{n}$.

More strictly, mass $M_{n}$ in (1.5) should be presented as

$$
M_{n}=n M_{0}+n_{L} M_{L},
$$

where $M_{0}$ and $M_{L}$ are the masses of single molecules of the substance forming the solid phase and of the medium of the system, $n_{L}$ is a number of medium molecules trapped in the volume and on the surface of the particle.

Then:

$$
n=\frac{v-n_{L} v_{0 L}}{v_{0}}
$$


where $v$ is a volume of a particle, $v_{0}$ and $v_{0 L}$ are volumes occupied by single molecules of the substance forming the solid phase and of the medium of the system inside the particle. Within the framework of the evaluation model, it is advisable to assume that the volume $v$ and surface area $s$ of each particle can be represented as functions of the effective size $l$ : when

$$
v>>n_{L} v_{0 L}
$$

we have:

$$
l_{M}=\gamma_{1} l, \quad s=\gamma_{2} l^{2}, \quad v=\gamma_{3} l^{3},
$$

where $\gamma_{1}, \gamma_{2}$ and $\gamma_{3}$ are shape factors, $l_{M}$ is maximum distance between points on the particle surface. A number of regularities in the behavior of a particle can be revealed by assuming that its shape is close to a sphere $\left(\gamma_{1}=1, \gamma_{2}=\pi\right.$, $\left.\gamma_{3}=\frac{\pi}{6}\right)$ or a cube $\left(\gamma_{1}=\sqrt{3}, \gamma_{2}=6, \gamma_{3}=1\right)$. For more accurate modeling, it is necessary to use experimentally determined shape factors.

If the particle has a plate shape, then it is advisable to assume that its volume is equal to $v=h s$, where $h$ is an effective thickness of the particle. If the particle is a chain of molecules, then $v=h^{2} l_{M}$. Therefore, in the general case, taking into account (1.7), (1.8) and (1.9), we have:

$$
n=\frac{\gamma_{\delta} h^{3-\delta} l^{\delta}}{v_{0}}
$$

where $v_{0}$ is the volume of one molecule, $\delta=1$ for chains of molecules, $\delta=2$ for plates, $\delta=3$ for spheres or cubes. We also assume that the shape parameters $\gamma_{\delta}$ doesn't depend on $l$.

The number of molecules $n$ constituting the particle is the main parameter, and so, it is of interest in the first turn the distribution functions of a dispersed substance by it. In the experiment, we obtain the integral distribution function depending on the particle's size $l$, for which a discrete set of values at points are measured. We denote the integral distribution function of aggregates of generation $k$ simulating (approximating) the experimental discrete distribution as $\theta_{k}(l)$ and the corresponding differential distribution function as $\phi_{k}(l)$.

We consider the two-component system which consists of the solvent or the carrier gas and the substance forming the solid phase. The system is restricted by the inert walls, which have the desired properties and transfer impacts from the system's surroundings. The number of molecules of the reagent and the number of molecules of the system medium are fixed: they are constant or vary in a specific manner. The system is considered as a set of homogeneous areas. The system is characterized by its volume, temperature, concentration of component at each point, pressure of the gas or of the liquid matter on the walls, and energies of various types is supplied in it. The laws of reflection of gas from the walls can be found in [12]. The dynamics of an elastic collision of two bodies has been studied by J. C. Maxwell [13].

The growth process of the aggregates of the type $k$ is determined by the frequency of collisions with particles of smaller generation than $k$. The reason for the growth of the particles is a long-ranged attraction between them: because of this, during the acts of the collision, they coalesce. The repulsion is short-ranged. The fragmentation has two causes - due to the excited state of the particle itself and because of external collisions with the carrier gas.

The elementary act of the formation of aggregates can be considered as the collision of two particles of any kind with their subsequent stay in contact for a sufficiently long time. The frequency of such acts can be represented as:

$$
\alpha=\alpha_{12} N_{1} N_{2} \exp \frac{-\varepsilon_{A}}{k T},
$$

where $\alpha_{12}$ is a frequency function associated with the probability of particles approaching until the contact, $N_{1}$ and $N_{2}$ are particle concentrations, $\varepsilon_{A}$ is an activation energy required to overcome the attraction of particles to the molecules of their environment. The termination of particle contact can be considered as an act of fragmentation of the aggregate. The fragmentation of aggregates is expediently described by the Arrhenius equation [11, 14, 15], introducing into consideration the activation energy of fragmentation $\varepsilon_{F}$.

The frequency functions of particles, which are volumes of molecules, can be proportional to $n^{1 / 3}, n^{2 / 3}$, or be independent on $n$. The first case means that the active centers are one-dimensional structure, in the second one the number of active centers is proportional to the square of the surface, and in the last case the number of active centers is a fixed number.

The question about the formation and about the size of the particles, starting from which the particle begins to grow rapidly, is a separate problem. The experiments demonstrate the existence of such size, and the problem is to construct a mathematical model which evaluates this size. At a certain particle size, the molecules in it are arranged due to the mutual attraction, and the probability of the fragmentation decreases. This particle has low porosity and obtains the certain shape. In the mathematical modeling, as the maximum of simplification, it is necessary to consider three forms of the particles which are the one-dimensional, two-dimensional and three-dimensional configurations. 
Based on the consideration of the three configurations, we can estimate their stability, which depends on the binding energy between molecules in the particle.

We'll consider only two parameters characterizing the particle of a nanodispersed substance: its mass and its potential energy, which we will account in the equations of the model.

The aim of our investigation is to determine the coefficients of the equations for the distribution function from ones, which are obtained in experiments. Thus, the first problem is to obtain this equations, the second one is to consider the classes of distribution functions of particles of a nanodispersed substance, reasonably approximating the experimental data obtained, and the third is to obtain relationships between the parameters of such functions and the coefficients of the equations.

The first problem that arises is the obtaining of correct equations describing the kinetic of a nanodispersed substance, and, hence, the derivation of the equations from each other: the consideration of the connection between the discrete and continuum equations, the transition from a description with multiple parameters to a reduced one. For instance, the derivation of equations on the particle size distribution function from the generalized Boltzmann-type equations for the distribution function of bodies by sizes and velocities of their centers of mass. The second problem was preliminarily considered in [11]. In the next paragraph, we begin to discuss the first one. A discrete distribution function is considered, and the equations for it is obtained. For particles consisting of less than $10^{2}-10^{3}$ molecules, the introduction of the continuum description is not advisable.

In Section 2 it is shown how from the discrete description the continuum equations of the Fokker-Planck type are obtained. In probability theory, the Fokker-Planck equation is called the differential Einstein-Kolmogorov equation [16]. Due to the parabolic nature of the obtained equation, it is also called the diffuse approximation.

In Section 3, we determine the coefficients of the considered kinetic equations of a nanodispersed substance by their stationary solution. However, in experiments, we obtain distribution functions which can't be the stationary solutions of the considered equations. They will continue disperse in the space of particles' sizes. We discuss such functions in the following paragraphs.

In Sections 4 and 5, we discuss the parameters and the classes of distribution functions of particles of a dispersed substance, approximating well the data obtained in the experiments.

In Section 6, we consider the exact solution of the diffuse approximation, which coincides with the proposed new approximating distribution function of particles of a dispersed substance at each moment of time and obtain the relationships between the parameters of approximating functions and the coefficients of the equations describing the kinetic of a dispersed substance.

\section{The Becker-Doring case and the continuum description of the kinetics of a dispersed substance}

Let us write the simplest kinetic equations for the evolution of the distribution function of particles of a dispersed substance: first, the discrete ones, and then the continuous ones (the equations of the Fokker-Planck type).

The equations for the coalescence of particles were first derived and studied for solutions by Smoluchowski [17] (without the fragmentation - the Smoluchowski case). If only one molecule can attach to or separate from another particle, then we have the Becker-Döring system of equations [18]. It describes the first stage of the aggregation process. During the formation of first generation aggregates, it can be assumed that their number is quite small, and therefore the formation of the secondary aggregates consisting of them can be neglected. Becker and Döring [18] have written an infinite system, but in the real physical problem numbers, $n$ is always bounded above: $n \leq m$, where $m$ is some natural number, whose value is determined by the properties of the system. The most rough estimate of $m$ obviously gives that $m \leq N_{0}$, where $N_{0}$ is the number of all molecules of the forming the phase substance in the system. In this case for the homogeneous system, we have the following system of equations for the evolution of the discrete distribution function of aggregates for the case $k=1$ and for single molecules $N(1, t)$ [18] (we'll skip the index $k=1$ in the following equations):

$$
\begin{gathered}
\frac{d N(1, t)}{d t}=-2\left(\alpha(1) N(1, t)^{2}-\beta(1) N(2, t)\right)-\sum_{i=2}^{m-1}(\alpha(i) N(1, t) N(i, t)-\beta(i+1) N(i+1, t)), \\
\frac{d N(n, t)}{d t}=[(\alpha(n-1) N(1, t) N(n-1, t)-\beta(n) N(n, t))+ \\
+(\beta(n+1) N(n+1, t)-\alpha(n) N(1, t) N(n, t))], n=2,3, \ldots, m-1, \\
\frac{d N(m, t)}{d t}=\alpha(m-1) N(1, t) N(m-1, t)-\beta(m) N(m, t),
\end{gathered}
$$

where $N(n, t)$ is a concentration (or a number) of the aggregates of the first generation consisting of $n$ molecules for $n=2,3, \ldots, m, N(1, t)$ is a concentration (or a number) of single molecules, $\alpha(n)$ is a frequency function (a cross 
section) of the coalescence of an original aggregate consisting of $n$ molecules with the single molecule, and $\beta(n)$ is a frequency of disintegration of a cluster consisting of $n$ molecules into the particle consisting of $(n-1)$ molecules and the single molecule.

But experiments and calculations on a computer require the discrete equations with time step, $\Delta t$, so we must write $\frac{\Delta N(n, t)}{\Delta t}$ instead of $\frac{d N(n, t)}{d t} . \Delta N(n, t)$ is a variation of $N(n, t)$ during time interval $\Delta t$. We must give definition of it through $N(n, t)$. If $\Delta N(n, t) \equiv N(n, t+\Delta t)-N(n, t)$, then we have the explicit time discretization of the system (2.1)-(2.3). For the system of equation (2.1)-(2.3) the $H$-theorem is fulfilled, but for the system with the explicit time discretization the $H$-theorem is not valid: it is proved in $[19,20]$ that the $H$-theorem is not fulfilled for the case of this system when only single molecules and dimers are considered. Also, it is valid for the implicit time discretization: when $\Delta N(n, t) \equiv N(n, t)-N(n, t-\Delta t)$ [20,21], and thus, we can't use the explicit time discretization for the computer simulations.

Instead the equation (2.1) one can write the conservation law of the number of all molecules of the forming the phase substance in the system $N_{0}$ :

$$
\sum_{n=1}^{m} n N(n, t) \equiv N_{0}
$$

where $N_{0}$ is constant.

Here, we have considered the discrete distribution function, which is the ordered set of numbers $N(n, t), n=$ $1,2, \ldots, m$. The equations (2.1), (2.3) are the boundary conditions for equations (2.2).

In the previous section we discuss a more general situation than one which is described by these systems, but in this case we have equations of the same form:

$$
\begin{gathered}
\Delta N_{k}(n, t)= \\
=\Delta t\left[\sum_{p=1}^{k-1}\left(\sum_{c \geq n_{p, 0}} \alpha_{k, p}(n-c, c) N_{p}(c, t) N_{k}(n-c, t)-\sum_{d \geq n_{p, 0}} \beta_{k, p}(n, d) N_{k}(n, t)\right)+\right. \\
\left.+\sum_{p=1}^{k-1}\left(\sum_{d \geq n_{p, 0}} \beta_{k, p}(n+d, d) N_{k}(n+d, t)-\sum_{c \geq n_{p, 0}} \alpha_{k, p}(n, c) N_{p}(c, t) N_{k}(n, t)\right)+W\right]
\end{gathered}
$$

for $n \geq n_{k, 0}$, where $n_{p, 0}$ is a minimum number of molecules in a particle of type $p, \Delta N_{k}(n, t)$ is as before a variation of the number of particles $N_{k}(n, t)$ of the type $k$ consisting of $n$ molecules during time interval $\Delta t, \alpha_{k, p}(n, c)$ is a frequency function (a section) of the coalescence of the particle of the generation $k$ consisting of $n$ molecules with the particle of the type $p$ consisting of $c$ molecules, $\beta_{k, p}(n, d)$ is a frequency of fragmentation of the particle of the kind $k$ consisting of $n$ molecules into the particle of the same kind consisting of $(n-d)$ ones and the particle of the generation $p$ consisting of $d$ molecules. Term $W$ in (2.5) takes into consideration that the number of particles of the kind $k$ can varies also due to the coalescence of two particles of generation $k-1$, because of a result of the reverse process, due to the coalescence of the particle of the type $k$ with particles of generation more or equal to $k$ and due to the fragmentation the particle of the kind $k$ from particles of generation more or equal to $k$.

The histograms, which get the experimenters, as a rule, are replaced by continuous functions. So it is necessary to use a continuum description. You can transit from a discrete distribution function and the system of equation (2.1)(2.3) to the continuum model by the introduction of the continuum distribution functions of particles of a nanodispersed substance $\psi_{k}(n, t)$, for which the accordance to the discrete distribution of particles of a nanodispersed substance is valid:

$$
\sum_{i=n_{k, 0}}^{n} N_{k}(i, t)=\int_{n_{k, 0}}^{n} \psi_{k}(\tilde{n}, t) d \tilde{n} .
$$

Further, we'll only consider the case $k=1$, so we'll skip the index $k=1$. The continuum problem is considered on the interval $\left[n_{1}, n_{2}\right]$. Here, $n_{1}$ and $n_{2}$ are the minimum and maximum number of molecules in the cluster, the growth of which can be considered as a continuous process. The minimum possible value is $n_{1} \approx 10^{2}$ [5], and $n_{2} \leq m$. The simple continuum analogy of (2.2) has the form:

$$
\frac{\partial \psi(n, t)}{\partial t}=(\alpha(n-1) N(1, t) \psi(n-1, t)-\beta(n) \psi(n, t))+(\beta(n+1) \psi(n+1, t)-\alpha(n) N(1, t) \psi(n, t)),
$$

where now $\alpha(n)$ and $\beta(n)$ are continuum functions.

The conservation law of the number of all molecules of the forming the phase substance in the system (2.4) is rewritten in the form:

$$
\sum_{n=1}^{n_{1}-1} n N(n, t)+\int_{n_{1}}^{n_{2}} n \psi(n, t) d n+\sum_{n=n_{2}+1}^{m} n N(n, t)=N_{0} .
$$


The equations (2.6), (2.7) is proved by that the substitution the sum of the $\delta$-functions: $\psi(n, t)=\sum_{i=1}^{n_{M}} N(i, t)$. $\delta(n-i)$, gives (2.2), (2.3). Indeed, the transition from (2.7) to (2.4) is obvious. Substituting in (2.6) the sum of the $\delta$-functions and integrating for each positive integer $n \in\left[n_{1}, n_{2}\right]$ the both parts of the obtained equation on the interval containing only one integer value of $n$, we obtain (2.2), (2.3) for this $n$.

Let us decompose the increments of the functions in (2.6) $\alpha(n) N(1, t) \psi(n, t)$ and $\beta(n) \psi(n, t)$ in the Taylor series and consider only two terms of the decomposition in the Taylor series. Then we obtain the equation of the Fokker-Planck type for the function $\psi=\psi(n, t)$, which has the form:

$$
\frac{\partial \psi}{\partial t}=-\frac{\partial}{\partial n} J[N(1, t), \psi]=-\frac{\partial}{\partial n}(G(n, t) \psi)+\frac{\partial^{2}}{\partial n^{2}}(D(n, t) \psi),
$$

where $J[N(1, t), \psi] \equiv G(n, t) \psi-\frac{\partial}{\partial n}(D(n, t) \psi), G(n, t) \equiv \alpha(n, t) N(1, t)-\beta(n, t)$, $D(n, t) \equiv \frac{\alpha(n, t) N(1, t)+\beta(n, t)}{2}$. So, $D(n, t)>0$.

Thus, $\alpha$ and $\beta$ are determined by the functions:

$$
\alpha(n, t)=\frac{G(n, t)+2 D(n, t)}{2 N(1, t)}, \quad \beta(n, t)=\frac{2 D(n, t)-G(n, t)}{2} .
$$

The problem (2.8) is solved on the interval $\left[n_{1}, n_{2}\right]$, and it is supplemented by the equations (2.2) for $2 \leq n \leq$ $n_{1}-1$ and for $n_{2}+1 \leq n \leq m$ (if $n_{2}<m$ ), by the conservation law of the number of all molecules of the forming the phase substance in the system (2.7) and by the boundary conditions.

The boundary conditions will be the relationship of the equality of flows of the continuum distribution function: $J[N(1, t), \psi(n, t)]$, and of the discrete one on the left and right bounds of the continuum distribution:

$$
\begin{aligned}
& \left.J[N(1, t), \psi(n, t)]\right|_{n=n_{1}}=\alpha\left(n_{1}-1, t\right) N(1, t) N\left(n_{1}-1, t\right)-\beta\left(n_{1}, t\right) N\left(n_{1}, t\right), \\
& \left.J[N(1, t), \psi(n, t)]\right|_{n=n_{2}}=\alpha\left(n_{2}, t\right) N(1, t) N\left(n_{2}, t\right)-\beta\left(n_{2}+1, t\right) N\left(n_{2}+1, t\right),
\end{aligned}
$$

where $N\left(n_{1}, t\right) \equiv \psi\left(n_{1}, t\right), N\left(n_{2}, t\right) \equiv \psi\left(n_{2}, t\right)$, if $n_{2}<m$, and

$$
\left.J[N(1, t), \psi(n, t)]\right|_{n=n_{2}}=0,
$$

if $n_{2}=m$.

If $n$ is not restricted from above $m \rightarrow+\infty$, then we have the boundary condition at the infinity:

$$
\lim _{n \rightarrow+\infty}\left(n^{\lambda}(\alpha(n, t) N(1, t) N(n, t)-\beta(n+1, t) N(n+1, t))\right) \rightarrow 0,
$$

for some $\lambda>1$. In this case the sum in the right side of (2.1) is finite, when $m \rightarrow+\infty$. If $n_{2}=m \rightarrow+\infty$, we have:

$$
\left.n^{\lambda} J[N(1, t), \psi(n, t)]\right|_{n \rightarrow+\infty} \rightarrow 0,
$$

The following system of equations is also considered (e.g., [8]): $N(n, t)=0$ for $2 \leq n \leq n_{0}-1\left(n_{0}>2\right)$. It accords to the simple assumption that besides the single molecules the particles, consisting of smaller than $n_{0}$ molecules, don't exist. The particles consisting from $n_{0}$ molecules give $n_{0}$ molecules, when they fragmentize. Let for simplicity $n_{1}=n_{0}, n_{2}=m \rightarrow+\infty$. $n_{0}$ may depend on time $t$. Then we have the equation (2.8) on the interval $\left[n_{0},+\infty\right)$ and the conservation law of the number of all molecules of the forming the phase substance in the system, which has the form:

$$
N(1, t)+\int_{n_{0}(t)}^{+\infty} n \psi(n, t) d n=N_{0} .
$$

In this case, we have the boundary conditions for the equation (2.8) on the right bound (2.14) and one on the left bound, which is obtained from (2.15).

In (2.1)-(2.3) the coalescence of particles due to pair collisions is assumed. This supposition is valid only for rarified gases. The experiments are described by the system of equations, which is more general, than (2.1)-(2.3): instead of terns $\alpha(n) N(1, t)$ we must write some unknown function $\tilde{\alpha}(n, N(1, t))$, which is a linear function: $\tilde{\alpha}(n, N(1, t))=\alpha(n) N(1, t)$, only for rarified gases. The equation of the Fokker-Planck type is obtained similarly. It has the form (2.8), where:

$$
G(n, t) \equiv \tilde{\alpha}(n, N(1, t))-\beta(n), \quad D(n, t) \equiv \frac{\tilde{\alpha}(n, N(1, t))+\beta(n)}{2} .
$$

So, $D(n, t)>0, \tilde{\alpha}$ and $\beta$ are determined by the functions:

$$
\tilde{\alpha}=G(n, t)+2 D(n, t), \quad \beta=2 D(n, t)-G(n, t) .
$$


The boundary conditions (2.14) and the conservation law of all molecules of the forming the phase substance in the system are the same.

Our further aim is to determine the coefficients of the equations by the stationary and non-stationary distribution functions of particles of a dispersed substance.

\section{The determination of coefficients of the kinetic equations of a dispersed substance by their stationary solution}

Let the frequency functions be nonzero.

It is obvious, that the stationary solution of (2.1)-(2.3): $N(n, t)=N_{e q}(n)$ ("eq" from "equilibrium"), $n=$ $1,2, \ldots, m$, satisfies the relationships:

$$
\alpha(n) N_{e q}(1) N_{e q}(n)=\beta(n+1) N_{e q}(n+1),
$$

for $n=1,2,3, \ldots, m-1$, and the conservation law (2.7). In (3.1) for the stationary solution $N_{e q}(n)$ the rate of the forward process (of the coalescence) equals the rate of reverse one (which is fragmentation) for each of such processes. For the physico-chemical kinetic equations such relationships are called the condition of detailed balance. From (3.1) and $N_{e q}(n+1)-N_{e q}(n) \equiv \Delta N_{1}(n)$ we have:

$$
\left(\alpha(n) N_{e q}(1)-\beta(n+1)\right) N_{e q}(n)=\beta(n+1) \Delta N_{1}(n) .
$$

From (3.1) we also obtain:

$$
N_{e q}(n)=\frac{N_{e q}^{n}(1) \prod_{j=1}^{n-1} \alpha(j)}{\prod_{j=2}^{n} \beta(j)} \quad \text { for } n=2,3, \ldots, m
$$

This relation indicates that $\alpha(n)$ and $\beta(n)$ is sufficient to determine the function $N_{e q}(n)$. Indeed, substituting (3.3) in the conservation law (2.4) we obtain the equation for calculation of $N_{e q}(1)$.

Also, we can solve the reverse problem: due to (3.1) we are able to find the ratios of coefficients $\frac{\beta(n+1)}{\alpha(n)}$, where $n=2,3, \ldots, m$, by the stationary solution. Thus, according to (3.1), for the system of equations (2.1)-(2.3), we can find the frequency function $\alpha(n)$ or $\beta(n)$ by the stationary solution, if we know another frequency function: $\beta(n)$ or $\alpha(n)$.

Let us consider the same issue for hybrid (discrete and continuous) problem: the equation (2.11) on the interval $\left[n_{1}, n_{2}\right]$, which is supplemented by the system of equations (2.2)-(2.3) for $n=2, \ldots, n_{1}-1$ and for $n=n_{2}+1, \ldots, m$ (if $n_{2}<m$ ), by the conservation law of the number of all molecules of the forming the phase substance in the system (2.7) and by the boundary conditions (2.10), (2.11) for $n_{2}<m$ or (2.10), (2.12) for $n_{2}=m$.

Let us denote the stationary solution of this problem as $N_{e q}(n)$ for $n=1,2, \ldots, n_{1}-1, n_{2}+1, \ldots, n_{M}$ and $\psi_{e q}(n)$ for $n \in\left[n_{1}, n_{2}\right]$.

From equations (2.2), (2.3) for $n=n_{2}+1, \ldots, m$ (if $n_{2}<m$ ) we obtain that the stationary solution satisfies the relationships of detailed balance (3.1) for $n=n_{2}, \ldots, m-1$. According to this and (2.11), for $n_{2}<m$ or due to (2.15) for $n_{2}=m$ :

$$
\left.J\left[N_{e q}(1), \psi_{e q}(n)\right]\right|_{n=n_{2}}=0 .
$$

A stationary solution of (2.8) is determined by the equation $J\left[N_{e q}(1), \psi_{e q}(n)\right]=C(t)$ for $n \in\left[n_{1}, n_{2}\right]$. According to (3.4) $C(t) \equiv 0$, and we have:

$$
J\left[N_{e q}(1), \psi_{e q}(n)\right] \equiv\left(\alpha(n) N_{e q}(1)-\beta(n)\right) \psi_{e q}(n)-\frac{d}{d n}\left(\left(\alpha(n) N_{e q}(1)+\beta(n)\right) \psi_{e q}(n)\right)=0
$$

for $n \in\left[n_{1}, n_{2}\right]$. (2.10) gives that $\alpha\left(n_{1}-1\right) N_{e q}(1) N_{e q}\left(n_{1}-1\right)-\beta\left(n_{1}\right) N_{e q}\left(n_{1}\right)=0$, and, therefore, from the equations (2.2) for $n=2, \ldots, n_{1}-1$ (if $n_{1}>2$ ) we obtain that the stationary solution satisfies the relationships of detailed balance (3.1) for $n=1, \ldots, n_{1}-2$.

Thus, the stationary solution satisfies the following hybrid system of equations: the relationships of detailed balance (3.1) for $n=1,2,3, \ldots, n_{1}-1, n_{2}, \ldots, m-1$, the equation (3.5) and the conservation law of the number of all molecules of the forming the phase substance in the system (2.7).

As before, we subsequently find the values of $N_{e q}(n)$ for $n=2, \ldots, n_{1}$ through the value of $N_{e q}(1)$ : the formula (3.3). Then we solve the equation (3.5) with initial data $N_{e q}\left(n_{1}\right)=\frac{N_{e q}^{n}(1) \prod_{j=1}^{n_{1}-1} \alpha(j)}{\prod_{j=2}^{n_{1}} \beta(j)}$ and find $N_{e q}(n)$ for $n \in\left[n_{1}, n_{2}\right]$ through the value of $N_{e q}(1)$. So, in particular, now we know $N_{e q}\left(n_{2}\right) \equiv \psi_{e q}\left(n_{2}\right)$ through the value of $N_{e q}(1)$. Then, we again use the relationships of detailed balance (3.1) for $n=n_{2}, \ldots, m-1$ and calculate the 
remaining values of $N_{e q}(n)$. Finally, we substitute all these values in (2.10) and find $N_{e q}(1)$. Thus, we find the stationary solution by the frequency functions.

Also we can solve the reverse problem. So, the following theorem is valid.

Theorem. For the system of equations (2.7), (2.8) and (2.2), (2.3) for $n=2, \ldots, n_{1}-1$ and for $n=n_{2}+1, \ldots, m$ (if $n_{2}<m$ ) with the boundary conditions (2.10), (2.11) for $n_{2}<m$ or (2.10), (2.12) for $n_{2}=m$ the frequency function $\alpha(n)$ or $\beta(n)$ for $n=1,2, \ldots, n_{1}-1, n_{2}+1, \ldots, n_{M}-1$ and for $n \in\left[n_{1}, n_{2}\right]$ can be calculated by the stationary solution, if we know another frequency function.

The proof. The frequency function is determined by the relationships of detailed balance (3.1) for $n=1,2,3, \ldots$, $n_{1}-1, n_{2}, \ldots, n_{M}-1$, and the equation (3.5) for $n \in\left[n_{1}, n_{2}\right]$. According to the condition of detailed balance we solve (3.5) with initial data $\beta\left(n_{1}\right)=\frac{\alpha\left(n_{1}-1\right) N_{e q}(1) N_{e q}\left(n_{1}-1\right)}{N_{e q}\left(n_{1}\right)}$, if we calculate $\beta(n)$, and with $\alpha\left(n_{2}\right)=$ $\frac{\beta\left(n_{2}+1\right) N_{e q}\left(n_{2}+1\right)}{\left(N_{e q}(1) N_{e q}\left(n_{2}\right)\right)}$, if we calculate $\alpha(n)$.

\section{The parameters of modeling distribution functions of particles of a nanodispersed substance}

A distribution function by sizes $\phi(l)$ is usually used. It is such function, that $\int_{l_{0}}^{l} \phi\left(l^{\prime}\right) d l^{\prime}$ gives the number of particles with sizes which are less than $l$ and more than $l_{0}$, where $l_{0}$ is the smallest particle's size. In equations, we consider the distribution function by the number of molecules constituting the particle $\psi(n)$ instead of $\phi(l)$, considering that these functions are related to the fact that $\int_{l_{0}}^{l} \phi\left(l^{\prime}, t\right) d l^{\prime}=\int_{n_{0}}^{n(l)} \psi(n, t) d n$, where $n(l)$ is a number of molecules constituting a particle with size $l$. The total number of particles is $N(t) \equiv \int_{l_{0}}^{l_{M}} \phi\left(l^{\prime}, t\right) d l^{\prime}=\int_{n_{0}}^{n_{M}} \psi(n, t) d n$, where $l_{M}$ is the biggest particle's size, $n_{M}$ is the largest number of molecules constituting a particle. The integral distribution functions $\theta(l)$ are also considered: $\theta(l) \equiv \frac{1}{N(t)} \int_{l_{0}}^{l} \phi\left(l^{\prime}, t\right) d l^{\prime}$. They are normalized to the total number of particles $N(t)$. Thus, they take values from zero to one.

In order to describe the particle size distribution of powders, basically, four formulas are used in practice [22]: the Gorden-Andreev [22, 23], the Rozin-Rammlar-Bennett [24], [22], the normal [25-29] or lognormal [25-28, 30-48] distributions. In other research [27,36,37], it is considered the issue that distributions with only two parameters are insufficient. Using of normal and lognormal distribution with $l_{0}=0$ and $l_{M} \rightarrow+\infty$ is inadvisable.

The Gorden-Andreev formula is the function with only two parameters:

$$
\theta(l)=A l^{q},
$$

which, generally speaking, is not an integral distribution function, as it tends to infinity when $l \rightarrow+\infty$, and integral distribution functions should to take values from zero to one. It is applied for small $l$.

The Rozin-Rammlar-Bennett formula is also the distribution function with only two parameters:

$$
\theta(l)=1-\exp \left(-A l^{q}\right)
$$

and is the prototype of the functions with tree and four parameters, which we'll consider further.

Let us note that the formula (4.1) is obtained as the first two terms of the decomposition in the Maclaurin series of the function (4.2). This indicates its applicability for small $l$.

Let $\theta_{e}(l)$ ("e" from "experiment") is the integral distribution function measured in the experiment for a discrete set of sizes of particles. $\theta_{e}(l)=\frac{N_{e}(l)}{N_{e}}$, where $N_{e}(l)$ is a number of measured particles with size less than $l, N_{e}$ is a total number of measured particles. We'll call such set of parameters for modeling functions, in which the minimum of the deviation of the function $\theta_{e}(l)$ from $\theta_{e}(l)$ is achieved, as the optimal set of parameters.

The parameters of the distribution function are the smallest particle's size $l_{0}$ (it corresponds to the minimum number of molecules in the cluster: $\left.n=n_{0}\right): \theta\left(l_{0}\right)=0$, the median value $l_{R}$ (it corresponds to $n=n_{R}$ ):

$$
\theta\left(l_{R}\right)=\frac{1}{N(t)} \int_{l_{0}}^{l_{R}} \phi\left(l^{\prime}, t\right) d l^{\prime}=\frac{1}{N(t)} \int_{n_{0}}^{n_{R}} \phi\left(n^{\prime}, t\right) d n^{\prime}=\frac{1}{2},
$$

and the maximum particle size $l_{M}: \theta\left(l_{M}\right)=1$. The value $l_{0}$ is from the interval $\left(0, l_{1}\right)$. And it is required that $\frac{l_{0}}{l_{1}} \approx 1$, because in the experiment particles with size smaller, than $l_{1}$, aren't registered. 


\section{The classes of modeling integral distribution functions of particles of a nanodispersed substance}

We considered the following classes of modeling integral distribution functions of particles of a nanodispersed substance:

$$
\theta(l)=1-\exp (-\omega(l)),
$$

where we took the following functions $\omega(l)$, containing the power parameter $(q)$ :

$$
\omega(l)=\omega_{1}(l)=a_{0}\left(Z_{1}(l)\right)^{q}, \quad \text { where } \quad a_{0}=\ln 2, \quad Z_{1}(l) \equiv \frac{l-l_{0}}{l_{R}-l_{0}},
$$

and

$$
\omega(l)=\omega_{2}(l)=a\left(Z_{2}(l)\right)^{q}, \quad \text { where } \quad Z_{2}(l) \equiv \frac{l-l_{0}}{l_{M}-l} .
$$

The optimal values of the parameter $q$ were fractional mainly in the range from one to three.

It is advisable to use the following class of functions:

$$
\theta(s)=1-\exp \left(-a_{1} Z_{3}(s)\left(1+a_{2} Z_{3}(s)\right)\right), \quad \text { where } \quad Z_{3}(s) \equiv \frac{s}{s_{0}-1} .
$$

Here, $a_{1}, a_{2}, s_{0}$ are parameters of the distribution function, $s=\gamma_{s} l^{2}$ is a square of a particle of a nanodispersed substance, $s_{0}=\gamma_{s} l_{0}^{2}$. (In the one-dimensional case (in the case of chains of molecules) the square of the image of the particle is proportional to $l$.) Equation (5.4) reasonably approximates the distribution functions of particles, which are surfaces of initial particles. In this case, $\delta=2$ in (1.10), and we can rewrite (5.4) in the following form:

$$
\theta(n)=1-\exp \left(-a_{1}\left(\frac{n}{n_{0}}-1\right)\left(1+a_{2}\left(\frac{n}{n_{0}}-1\right)\right)\right) .
$$

So, according (1.2) we have the classes of functions:

$$
\theta(l)=1-\exp \left(-a_{1}\left(\frac{l^{\delta}}{l_{0}^{\delta}}-1\right)\left(1+a_{2}\left(\frac{l^{\delta}}{l_{0}^{\delta}}-1\right)\right)\right)
$$

where $\delta=1,2,3$ respectively for chains, surfaces and volumes of initial particles.

We checked by calculations on the computer that the considered approximating functions describe well the experimental distribution functions of particles of nanodispersed substances [49-52].

\section{The relationships between parameters of approximating distribution functions of particles of a dispersed substance and the coefficients of the equation of the Fokker-Planck type describing their kinetics}

Now we try to connect the simple phenomenological formulas of Section 5 with the equations of the FokkerPlanck type from Section 2.

The equation of the Fokker-Planck type has the form (2.8). The equation (2.8) is considered in the region $n \in$ $\left[n_{0},+\infty\right), t \in\left[t_{0},+\infty\right)$. When $n<n_{0}$, the distribution function is equal to zero. If the functions $G$ and $D$ depend only on time - it is the case of fixed number of active aggregation centers on the surface of the particle, then (2.8) has the form:

$$
\frac{\partial \psi}{\partial t}=-\frac{\partial}{\partial n} J\left[N_{1}, \psi\right]=-G(t) \frac{\partial \psi}{\partial n}+D(t) \frac{\partial^{2} \psi}{\partial n^{2}},
$$

here, $J\left[N_{1}, \psi\right] \equiv-G(t) \psi+D(t) \frac{\partial \psi}{\partial n}$. For Equation (6.1) after the substitution [53 (p. 309)]:

$$
\tau=\tau(t)=\int_{t_{0}}^{t} D(x) d x+A, \quad z=z(n, t)=n-\int_{t_{0}}^{t} G(x) d x+B,
$$

where $A$ and $B$ are constants, gives the equation for the function $\tilde{\psi}(z, \tau) \equiv \psi(n, t)$ :

$$
\frac{\partial \tilde{\psi}}{\partial \tau}=\frac{\partial^{2} \tilde{\psi}}{\partial z^{2}}
$$

Let us consider in the following exact solution of (6.3) (see, e.g., [53 (p. 233)]):

$$
\tilde{\psi}(z, \tau)=C \frac{z}{\tau^{3 / 2}} \exp \left(-\frac{z^{2}}{4 \tau}\right) .
$$


In order, $\tau>0$, due to (6.2) the value of $A$ should be positive (here we use that $D(t)>0$ ). The function (6.4) is nonnegative, if and only if $z \geq 0$. So, according to (6.2) for any $t \in\left[t_{0},+\infty\right)$ :

$$
B \geq \int_{t_{0}}^{t} G(x) d x-n_{0}
$$

The condition of normalization gives the total number of all particles $N(t)$ at any fixed moment of time $t$, and due to (6.4) has the form:

$$
N(t)=\int_{n_{0}}^{+\infty} \psi(n, t) d n=\int_{z\left(n_{0}, t\right)}^{+\infty} \tilde{\psi}(z, \tau) d z=\frac{2 C}{\sqrt{\tau}} \exp \left(-\frac{\left(z\left(n_{0}, t\right)\right)^{2}}{4 \tau}\right) .
$$

From (6.4) and (6.6), we have:

$$
\theta=\frac{1}{N(t)} \int_{n_{0}}^{n} \psi(n, t) d n=\frac{1}{N(t)} \int_{z\left(n_{0}, t\right)}^{z(n, t)} \tilde{\psi}\left(z^{\prime}, \tau\right) d z^{\prime}=1-\exp \left(\frac{\left(z\left(n_{0}, t\right)\right)^{2}}{4 \tau}\right) \exp \left(-\frac{(z(n, t))^{2}}{4 \tau}\right) .
$$

Let us rewrite (5.5) in the form:

$$
\theta(n)=1-\exp \left(\frac{\left(n_{0}-b_{1}\right)^{2}}{4 b_{2}}\right) \exp \left(-\frac{\left(n-b_{1}\right)^{2}}{4 b_{2}}\right)
$$

where $b_{1}=n_{0}\left(1-\frac{1}{2 a_{2}}\right), b_{2}=\frac{n_{0}^{2}}{4 a_{1} a_{2}}$.

So, due to (6.2) we have the same function in (6.8) as in (6.7), if

$$
b_{1}(t)=n-z(n, t)=\int_{t_{0}}^{t} G(x) d x-B, \quad b_{2}(t)=\tau(t)=\int_{t_{0}}^{t} D(x) d x+A,
$$

We can check by the substitution, that the functions from the class (6.8) are solutions of (6.1), if and only if the relationships (6.9) are valid.

The conditions (6.5) and $\tau>0$ are fulfilled, if for any $t \in\left[t_{0},+\infty\right) b_{1}(t) \leq n_{0}(t), b_{2}(t)>0$. So, $a_{1}(t)$ and $a_{2}(t)$ should be positive.

We'll now strive to express the frequency functions $G$ and $D$ through the parameters of the simple phenomenological formulas of Section 5. From (6.9), we obtain $b_{1}\left(t_{0}\right)=-B, b_{2}\left(t_{0}\right)=A$, and:

or:

$$
\int_{t_{0}}^{t} G(x) d x=b_{1}(t)-b_{1}\left(t_{0}\right), \quad \int_{t_{0}}^{t} D(x) d x=b_{2}(t)-b_{2}\left(t_{0}\right),
$$

$$
G(t)=\frac{d b_{1}(t)}{d t}, \quad D(t)=\frac{d b_{2}(t)}{d t}
$$

According to the definition (2.16) of $G(t)$ and $D(t)$, they depend upon $t$ only through the temperature $T(t)$ and the concentration of single molecules $N(1, t): G(t)=g(T(t), N(1, t)), D(t)=d(T(t), N(1, t))$. If $T(t)$ and $N(1, t)$ are constant or their variations during time interval $[t, t+\Delta t]$ are negligible, then according to (6.10) by two measurements in the moments of time $t$ and $t+\Delta t$ we can find the values of $G=g\left(T, N_{1}\right)$ and $D=d\left(T, N_{1}\right)$ for the given temperature $T$ and the concentration of single molecules $N(1)$ :

$$
\begin{gathered}
G=\frac{b_{1}(t+\Delta t)-b_{1}(t)}{\Delta t}=\left(n_{0}(t+\Delta t)\left(1-\frac{1}{2 a_{2}(t+\Delta t)}\right)-n_{0}(t)\left(1-\frac{1}{2 a_{2}(t)}\right)\right) / \Delta t, \\
D=\frac{b_{2}(t+\Delta t)-b_{2}(t)}{\Delta t}=\frac{1}{4}\left(\frac{n_{0}^{2}(t+\Delta t)}{a_{1}(t+\Delta t) a_{2}(t+\Delta t)}-\frac{n_{0}^{2}(t)}{a_{1}(t) a_{2}(t)}\right) / \Delta t .
\end{gathered}
$$

Varying system's condition (considering different values of $T$ and $N(1, t)$ ) and calculating in each case values of $G=g(T, N(1))$ and $D=d(T, N(1))$ according to (6.11) we can find functions $g(T, N(1))$ and $d(T, N(1))$.

These formulas (6.11) connect the phenomenology of Section 5 with the Fokker-Planck type equation of Section 2. 
Let us note, that we vary the value of $N(1)$ in order to find the function $\tilde{\alpha}(N(1))$ in $(2.17)$. If we have a rarified gas, then we can assume the pair interaction. In this case, we can calculate $\alpha$ and $\beta$ according (2.9), where $N(1)$ is calculated according (2.15). In this case, we need not vary $N(1)$, and we can even calculate the frequency function without the condition that $N(1, t)$ is constant during time interval $[t, t+\Delta t]$.

We can consider other approximating functions instead of (5.5). For example, we can obtain the relationships between the parameters of normal distributions by $n$ with $n \in\left[n_{0},+\infty\right)$ and the coefficients of the equation of the Fokker-Planck type (6.1) (the case, when $G$ and $D$ depend only on time). The choice of which approximating class of functions (or which solution of the equation of the Fokker-Planck type) is realized depends upon the law of varying of the number of particles with minimum size $n_{0}$, which determines the initial data and the boundary condition on the left bound of the continuum distribution.

We have two one-dimensional functions in the coefficients of (6.1): $G(t)$ and $D(t)$. In order to determine these, we need at least two independent parameters in modeling class of functions depending upon time. The further development of the solving of this problem is to consider the case, when $G$ and $D$ depend upon $n$ with the number of parameters depending upon time more or equal to the number of one-dimensional functions in the coefficients of (2.8).

Thus, by two measurements of the distribution function of particles of a nanodispersed substance, we have learned to find the frequency functions, in the case when the temperature $T$ and the concentration of single molecules of the forming the phase substance $N(1)$ are constant or their relative variations is negligible. Varying system's condition, we can find frequency functions in dependence on $T$ and $N(1)$.

\section{Discussion of the results}

In the present paper, we considered the connection between the discrete equations describing the kinetics of particles of a dispersed substance and the continuum one, in the case when only single molecules integrate with or fragmentize from the particles, i.e. in the Becker-Döring case: equations (2.1)-(2.3). We obtained hybrid systems of equations, which have both discrete and continuum parts.

We considered the basic known simulating (approximating) distribution functions (formulas (4.1)-(4.6)) and new ones and their parameters. The exact solutions of the equation of the Fokker-Planck type, which gives distribution functions of particles of a nanodispersed substance, are found, and we learned to find frequency functions by the parameters of the modeling distribution functions.

Let us note that the consideration of the $H$-theorem for nonlinear systems with discrete time, in particular, even for the Becker-Döring system of equations, becomes an extremely important problem as the computer simulation has a significance in the solution of fundamental problem of the creating of new materials. In the linear case, the transition from continuous time to discrete gives the transition from a Markov process to a Markov chain and the $H$-theorem is valid and studied (see [54] and references in it, [55]). In the nonlinear case, for explicit time discretization it is fulfilled in rare cases [19,20] and for the implicit one, it is investigated in [20,21]. The diffuse approximation is widely used for the modeling of crystallization processes of a dispersed substance [5-8]. Therefore, the consideration of the $H$-theorem for it is of interest.

The equation of the Fokker-Planck type allows one to understand, to explain and even, if the initial information is enough, to predict the behaviors of the experimental distribution functions by properties of particles of a dispersed substance.

All approximating distribution functions considered in the present paper were single humped. But, of course, it is required not only such functions for practical problems: for example, with more than one extremum [56,57].

We have considered the distribution function of particles of a dispersed substance by the number of constituting of them molecules, because it is the basic parameter. Sometimes other parameters may be also very essential in a counterbalance to the distribution by sizes. The simplest distribution function by this parameter is such, when we take into account only monomers and dimers. It may be used for some practical problems. The state of each particle is characterized by many parameters. Even dimers are such, and one can consider the distribution function by these parameters [58]. And, of cause, very often, it is needed to investigate the distribution function by sizes and other parameters with its value, which is not only number or concentration of particles [29]. In these cases the distribution functions can have interesting plots [29]. And certainly, distribution functions of single initial particles (molecules, atoms, etc.) or nucleation centers are also considered [59].

It turned out that three-parameter functions of the type (5.6), which are more informative than the commonly used normal and log-normal distributions, and also the distributions (4.1), (4.2), have become available for determining. The availability of three-parameter distribution functions is indicated, in particular, by the data published in [52] about variations in the properties of nano-vesicles - niosomes, when they are heated to different temperatures. 


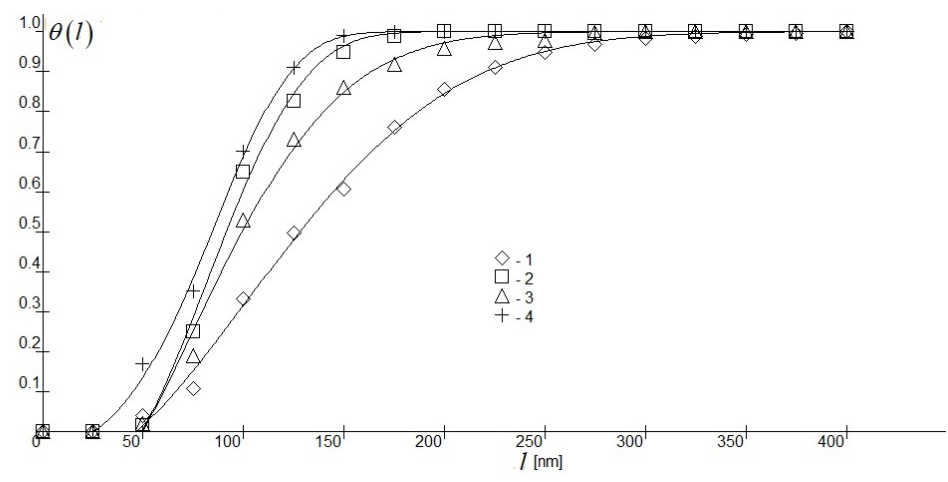

FIG. 1. Integral distribution functions of particle size in niosomal dispersions after heating at $303 \mathrm{~K}$ (1), $313 \mathrm{~K}(2), 323 \mathrm{~K}(3)$ and $333 \mathrm{~K}(4)$

TABLE 1. Distribution parameters of distribution functions (7.1) of particle size in niosomal dispersions after heating to temperatures of $303 \mathrm{~K}(1), 313 \mathrm{~K}(2), 323 \mathrm{~K}(3)$ and $333 \mathrm{~K}$ (4)

\begin{tabular}{|c|c|c|c|c|}
\hline Temperature & $303 \mathrm{~K}(1)$ & $313 \mathrm{~K}(2)$ & $323 \mathrm{~K}(3)$ & $333 \mathrm{~K}(4)$ \\
\hline$l_{0}, \pm 0.1 \mathrm{~nm}$ & 46.6 & 49.7 & 50 & 25.1 \\
\hline$a_{1}, \pm 0.01$ & 0.105 & 0.24 & 0.234 & 0.04 \\
\hline$a_{2}, \pm 0.001$ & 0,001 & 0,087 & 0,001 & 0,058 \\
\hline
\end{tabular}

Processing of these data showed that the studied niosomes had size distributions (Fig. 1) in accordance with the distribution function of the type (5.6), namely:

$$
\theta(l)=1-\exp \left(-a_{1} Z(l)\left(1+a_{2} Z(l)\right)\right),
$$

where $Z(l) \equiv \frac{l^{2}}{l_{0}^{2}}-1, a_{1}, a_{2}, l_{0}$ are distribution parameters.

When heated, niosomes were compressed while retaining the three-parameter function $\theta(l)$, and it cannot be ruled out that when heated at $T=333 \mathrm{~K}$, they were transformed into a heat-resistant state characterized by a parameter $l_{0}=25 \mathrm{~nm}$. It is also possible that additional information on the kinetics of such a transition can be extracted from the functions $a_{1}(T)$ and $a_{2}(T)$.

The distribution functions retain their informative value when the system transitions to an equilibrium state, as indicated by equations (3.1), containing parameters $\alpha(n)$ and $\beta(n)$. According to these relations, particles of different sizes can be present in the equilibrium system. The theorem of Section 3 indicates that it is possible to use data on the equilibrium distribution function to determine the kinetics for the formation and fragmentation of aggregates.

Relations (3.1) and (7.1) characterize the morphological diversity of dispersed systems, the distribution of particles by properties of which preserves information about the kinetics of the processes occurring in the systems.

\section{Acknowledgment}

This research was financially supported by the Ministry of Education and Science of the Russian Federation on the program to improve the competitiveness of Peoples' Friendship University of Russia (RUDN-University) "5-100" among the world's leading research and educational centers in 2016-2020.

\section{References}

[1] Maxwell J.C. On the Dynamical Theory of Gases. Philosophical Magazine, 1868, 4 ser, 35(235), P. 141-144.

[2] Boltzmann L. Weitere Studien über das Wärmegleichgewicht unter Gasmolekülen. Wien. Ber., 1872, 66, P. 275-370; Wissenschaftliche Abhandlungen, Barth, Leipzig, 1909, 1, P. 316-402.

[3] Konyakhin S.V, Sharonova. L.V., Eidelman. E.D. Labeling detonation nanodiamond suspensions using the optical methods. Tech. Phys. Lett., 2013, 39(3), P. 244-247.

[4] Aleksenskii A.E., Shvidchenko A.V., Eidel'man E.D. The applicability of dynamic light scattering to determination of nanoparticle dimensions in sols. Tech Phys Lett., 2012, 38(12), P. 1049-1052.

[5] Melikhov I.V. Physico-chemical evolution of solids. Binom, Moscow, 2006 (in Russian). 
[6] Melikhov I.V. Evolutional approach to creation of nanostructures. Nanosystems: Physics, Chemistry, Mathematics, 2010, 1(1), P. 148-155 (in Russian).

[7] Melikhov I.V., Mikheev N.B., Kulyukhin S.A., and Kozlovskaya E.D. Morphological Memory of Dispersed Solid Phases. Colloid Journal, 2001, 63(6), P. 738-745.

[8] Melikhov I.V., Rudin V.N., Kozlovskaya E.D., Adzhiev S.Z., Alekseeva O.V. Morphological memory of polymers and their use in developing new materials technology. Theoretical Foundations of Chemical Engineering, 2016, 50(3), P. 260-269.

[9] Eliseev A.A., Lukashin A.V. Functional nanomaterials. Under the editorship of Yu.D. Tretyakov. Fizmatlit, Moscow, 2010 (in Russian).

[10] Bayes Thomas, Price Thomas. An Essay towards solving a Problem in the Doctrine of Chance, By the late Rev. Mr. Bayes communicated by Mr. Price, in a letter to John Canton, M.A. and F.R.S. Phylocophical Transactions of the Royal Society of London, 1963, 53, P. 370-418.

[11] Adzhiev S.Z., Vedenyapin V.V., Volkov Yu.A., Melikhov I.V. Generalized Boltzmann-Type Equations for Aggregation in Gases. Computational Mathematics and Mathematical Physics, 2017, 57(12), P. 2017-2029.

[12] Batishcheva J.G. On the motion of solid in gas reacting with its surface. Derivation of the dynamical system. Keldysh Institute preprints, 2003, 18, 21 p. (in Russian).

[13] Maxwell J.C. Illustration of the Dynamical Theory of Gases. Philosophical Magazine, 1860, 1, P. $377-409$.

[14] Arrhenius S. Über die Reaktionsgeschwindigkeit bei der Inversion von Rohrzucker durch Säuren. Z. physik. Chemie (Leipzig), 1889, 4, P. 226-248.

[15] Stiller W. Arrhenius Equation and Non-Equilibrium Kinetics, 100 Years Arrhenius Equation. BSB B. S. Teubner Verlagsgesellschaft, Leipzig, 1989.

[16] Kolmogorov A.N. On the analytic methods in probability theory. Russian Mathematical Surveys, 1938, 5, P. 5-41 (in Russian).

[17] Smoluchowski M. Versuch einer mathematischen Theorie der Koagulationskinetik kolloider Losungen. Z. Phys. Chem., 1917, 92, P. 129-168.

[18] Becker R., Döring W. Kinetische Behandlung der Keimbildung in ubersattingten Dampfer. Ann. Phys., 1935, 24, P. 719-752.

[19] Adzhiev S.Z., Melikhov I.V., Vedenyapin V.V. The H-theorem for the physico-chemical kinetic equations with explicit time discretization. Physica A: Statistical Mechanics and its Applications, 2017, 481, P. 60-69.

[20] Adzhiev S., Melikhov I., Vedenyapin V. The H-theorem for the chemical kinetic equations with discrete time and for their generalizations. Journal of Physics: Conference Series, 2017, 788(1), P. 012001.

[21] Adzhiev S.Z., Melikhov I.V., Vedenyapin V.V. The H-theorem for the physico-chemical kinetic equations with discrete time and for their generalizations. Physica A: Statistical Mechanics and its Applications, 2017, 480, P. 39-50.

[22] Kousov P.A. The foundations of the analysis of disperse composition of industrial dusts and crushed materials. Chemistry, Leningrad, 1987 (in Russian).

[23] Andreev S.E. On the formulas of mean diameter. Mining journal, 1951, 11, P. 32-36 (in Russian).

[24] Rozin P., Rammlar E. Die Kornzusammensetzung des Mahlgutes im Lichte der Wahrscheinlichkeitslehre. Kolloid Zeitschrift, 1964, 67(1), P. 16-26.

[25] Fannin P.C., Charles S.W. AC susceptibility measurements in the investigation of aggregation in magnetic fluids. Physica Polonica A, 2000, 97(3), P. 591-594.

[26] Dahi S.R., Clelland R., Hrenya C.M. The effects of continuous size distributions on the rapid flow of inelastic particles. Physics of Fluids, 2002, 14(6), 1972.

[27] Vargas-Ubera J., Aguilar J.F., Gale D.M. Reconstruction of particle-size distributions from light-scattering patterns using three inversion methods. Applied Optics, 2007, 46(1), P. 124-132.

[28] Nosov V.V. Remote technique for simultaneous measurement of the particles' velocity and size distribution function. Atmospheric and oceanic optics, 1996, 9(1), P. 56-59.

[29] Bachina A., Ivanov V.A., Popkov V.I. Peculiarities of $\mathrm{LaFeO}_{3}$ nanocrystals formation via glycine-nitrate combustion. Nanosystems: Physics, Chemistry, Mathematics, 2017, 8(5), P. 647-653.

[30] Kolmogorov A.N. On the logarithmically normal distribution law of particle sizes under fragmentation. Dokl. Akad. Nauk SSSR, 1941, 31(2), P. 99-101 (in Russian).

[31] Aitchison J., Brown J.A.C. The Lognormal Distribution. Cambridge Univ. Press, 1963.

[32] Dacey M.F., Krumbein W.C. Models of breakage and selection for particle size distributions. Journal of the International Association for Mathematical Geology, 1979, 11(2), P. 193-222.

[33] Cooper D.W. On the products of lognormal and cumulative lognormal particle size distributions. Journal of Aerosol Sciences, 1982, 13(2), P. 111-120.

[34] Melik D.H., Fogler H.S. Turbidimetric determination of particle size distributions of colloidal systems. Journal of Colloid and Interface Science, 1983, 92(1), P. 161-180.

[35] LeBlanc S.E., Fogler H.S. Dissolution of powdered minerals: the effect of polydispersity. AIChE Journal, 1989, 35(5), P. 865-868.

[36] Buchan G.D., Grewal K.S., and Robson A.B. Improved models of particle-size distribution: an illustration of model comparison techniques. Soil Sci. Soc. Am. J., 1993, 57, P. 901-908.

[37] Wagner L.E., Ding D. Representing aggregate size distributions as modified lognormal distributions. Transactions of the ASAE, 1994, 37(3), P. 815-821.

[38] Chen Yongcheng, Zhang Yuanhui, Barber E.M. A new mathematical model of particle size distribution. Prairie Swine Centre Annual Research Report, 1994, P. 76-79.

[39] Chaiken J., Goodisman J. Application of fractals and kinetic equation in modelling cluster and ultrafine particle size distributions. NanoStructured Materials, 1995, 5(2), P. 225-231.

[40] Jonasz M., Fournier G. Approximation of the size distribution of marine particles by a sum of log-normal functions. Limnol. Oceanogr., 1996, 41(4), P. 744-754.

[41] Kiss L.B., Söderlund J., Niklasson G.A., Granqvist C.G. New approach to the origin of lognormal size distributions of nanoparticles. Nanotechnology, 1999, 10(1), P. 25-28.

[42] Selivanov V.N., Smyslov E.F. X-ray determination of the particle-size lognormal distribution in nanocrystalline materials. Industrial Laboratory, 2000, 66(11), P. 718-725. 
[43] Popa N.C., Balzar D. An analytical approximation for a size-broadened profile given by the lognormal and gamma distributions. Journal of Applied Crystallography, 2002, 35, P. 338-346.

[44] Ždímal V., Brabec M., Wagner Zd. Comparison of two approaches to modeling atmospheric aerosol particle size distributions. Aerosol and Air Quality Research, 2008, 8(4), P. 392-410.

[45] Rajesh T.A. Software Package for Aerosol Size Distribution. International Journal of Scientific Engineering and Technology, 2013, 2(4), P. 249-255.

[46] Hossain M.A., Mori Sh. Determination of particle size distribution of used black tea leaves by scanning electron microscope. Dhaka Univ. J. Sci., 2013, 61(1), P. 111-115.

[47] Desmond K.W. and Weeks E.R. Influence of particle size distribution on random close packing of spheres. Physical Review E, 2014, 90, P. 022204.

[48] Gao M., Xia Q., Akwe A.W. et al. Self-preserving lognormal volume-size distributions of starch granules in developing sweetpotatoes and modulation of their scale parameters by a starch synthase II (SSII). Acta Physiologiae Plantarum, 2016, 38(11), Article: 259.

[49] Qiao Yongsheng, Shen Yongsheng Lashes, Wu Meixia, Guo Yong, Meng Shuangming. A novel chemical synthesis of bowl-shaped polypyrrole particles. Materials Letters, 2014, 126, P. 185-188.

[50] Azmeera Venkanna, Rastogi Pankaj Kumar, Adhikary Pubali, Ganesan Vellaichamy, Krishnamoorthi S. Synthesis, characterization and cyclic voltammetric study of copper(II) and nickel(II) polymer chelates. Carbohydrate Polymers, 2014, 110, P. 388-395.

[51] Liu Junli, Ma Jianzhong, Bao Yan, Wang John, Zhu Zhenfeng, Tang Huiru, Zhang Limin. Nanoparticle morphology and film-forming behavior of polyacrylate/ZnO nanocomposite. Composites Science and Technology, 2014, 98, P. 64-71.

[52] Diskaeva E.I., Vecher O.V., Bazikov I.A., Vakalov D.S. Particle size analysis of niosomes as a function of temperature. Nanosystems: Physics, Chemistry, Mathematics, 2018, 9(2), P. 290-294.

[53] Polyanin A.D., Zaitsev V.F. Handbook on the differential equations with partial derivatives. Exact solutions. Intern. education program, Moscow, 1996 (in Russian).

[54] Adzhiev S.Z., Vedenyapin V.V. Time averages and Boltzmann extremals for Markov chains, discrete Liouville equations, and the Kac circular model. Comput. Math. Math. Phys., 2011, 51(11), P. 1942-1952.

[55] Vedenyapin V.V., Adzhiev, S.Z. Entropy in the sense of Boltzmann and Poincaré, Russian Math. Surveys, 2014, 69(6), P. $995-1029$.

[56] Almjasheva O.V., Gusarov V.V. Metastable clusters and aggregative nucleation mechanism. Nanosystems: Physics, Chemistry, Mathematics, 2014, 5(3), P. 405-416.

[57] Kuni F.M., Rusanov A.I. Statistical theory of aggregative equilibrium. Theoretical and Mathematical Physic, 1970, 2(2), P. $192-206$.

[58] Moliver S.S. Modeling chemisorption of carbon dimer at (8,0) nanotube. Nanosystems: Physics, Chemistry, Mathematics, 2017, 8(5), P. 641646.

[59] Durnev M.A., Eidelman E.D. Distribution of supercritical nucleation centers during the crystallization process in the presence of a flow as illustrated by the example of boron carbide-reinforced aluminum. Nanosystems: Physics, Chemistry, Mathematics, 2017, 8(3), P. 360-364. 